\title{
Successful Ablation with a Multipolar Mapping Catheter for Swallowing-induced Atrial Tachycardia
}

\author{
Naoaki Onishi ${ }^{1}$, Kazuaki Kaitani ${ }^{1}$, Kenji Yasuda ${ }^{2}$, Sousuke Sugimura ${ }^{2,3}$, Miyako Imanaka ${ }^{1}$, \\ Maiko Kuroda ${ }^{1}$, Shunsuke Nishimura ${ }^{1}$, Yusuke Takahashi ${ }^{1}$, Yusuke Yoshikawa ${ }^{1}$, \\ Masashi Amano ${ }^{1}$, Sari Imamura ${ }^{1}$, Yodo Tamaki ${ }^{1}$, Soichiro Enomoto ${ }^{1}$, Makoto Miyake ${ }^{1}$, \\ Toshihiro Tamura ${ }^{1}$, Hirokazu Kondo ${ }^{1}$, Chisato Izumi ${ }^{1}$ and Yoshihisa Nakagawa ${ }^{1}$
}

\begin{abstract}
We herein report a case of a 52-year-old woman who presented with a history of recurrent palpitations that occurred during swallowing solid food. On a Holter electrocardiogram, paroxysmal atrial tachycardias (PATs) were detected while eating. We mapped the right atrium (RA) with a multipolar mapping catheter while she swallowed a rice ball and it was revealed that the earliest endocardial breakthrough was on the anterior septal side near the superior vena cava junction of the RA. We successfully eliminated PAT at both the site in the RA and the adjacent right superior pulmonary vein ostium. After ablation, no PAT was documented while eating.
\end{abstract}

Key words: swallowing-induced atrial tachycardia, multipolar mapping catheter, PentaRay ${ }^{\circledR}$ NAV catheter, radiofrequency catheter ablation

(Intern Med 55: 2423-2427, 2016)

(DOI: 10.2169/internalmedicine.55.6418)

\section{Introduction}

Swallowing-induced atrial tachycardia (SIAT) is a rare disease. It is reported that the prevalence of SIAT is only $0.6 \%$ (1). Some mechanisms of SIAT have been suggested; for instance, it can be due to mechanical stimulation of the left atrium (LA) by a distended esophagus (2) or a neural mechanism (i.e., vasovagal reflexes and/or adrenergic reflexes) originating from the esophagus $(3,4)$. However, the precise mechanism has not yet been clarified. Furthermore, SIAT is detected only by swallowing; therefore, it is difficult to map the precise site of the earliest activation of SIAT during an electrophysiological study (EPS). We herein report a case of successful catheter ablation with a multipolar mapping catheter (PentaRay ${ }^{\circledR}$ NAV catheter, Biosense Webster, Diamond Bar, USA) for SIAT.

\section{Case Report}

A 52-year-old woman who presented with a 6-month history of recurrent palpitations occurring during swallowing solid food was referred to our institution and had no palpitations while drinking liquids. She had diabetes mellitus, a history of gastroduodenal ulcers, and had received medical therapy.

On a 12-lead electrocardiogram (ECG), paroxysmal atrial tachycardia (PAT) was repeatedly detected while eating (Fig. 1). On the ECG, the p wave polarity of PAT was positive in aVL and the inferior leads, and negative in V1, which indicated that the origin of PAT was the upper right atrium (RA) $(5,6)$. Upper gastrointestinal endoscopy revealed no abnormal findings. PAT was repetitively observed only while swallowing the scope, however, no arrhythmias occurred when the scope was passed through the esophagus. PAT became refractory with the administration of a beta-

${ }^{1}$ Division of Cardiology, Tenri Hospital, Japan, ${ }^{2}$ Department of Clinical Laboratory, Tenri Hospital, Japan and ${ }^{3}$ Division of Cardiology, Department of Medicine, Faculty of Medicine, Kinki University, Japan

Received for publication August 19, 2015; Accepted for publication January 5, 2016

Correspondence to Dr. Naoaki Onishi, n.onishi@kuhp.kyoto-u.ac.jp 


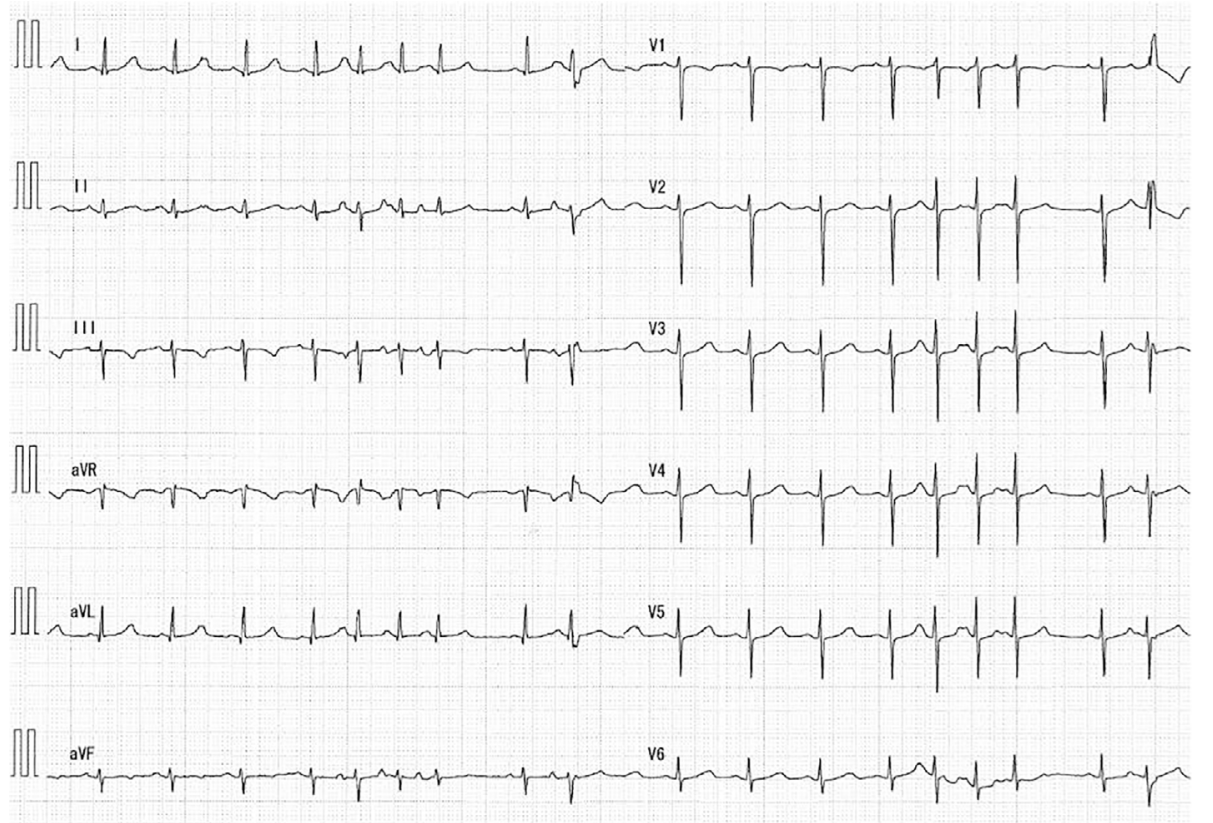

Figure 1. A 12-lead electrocardiogram of a paroxysmal atrial tachycardia detected only after swallowing solid food.
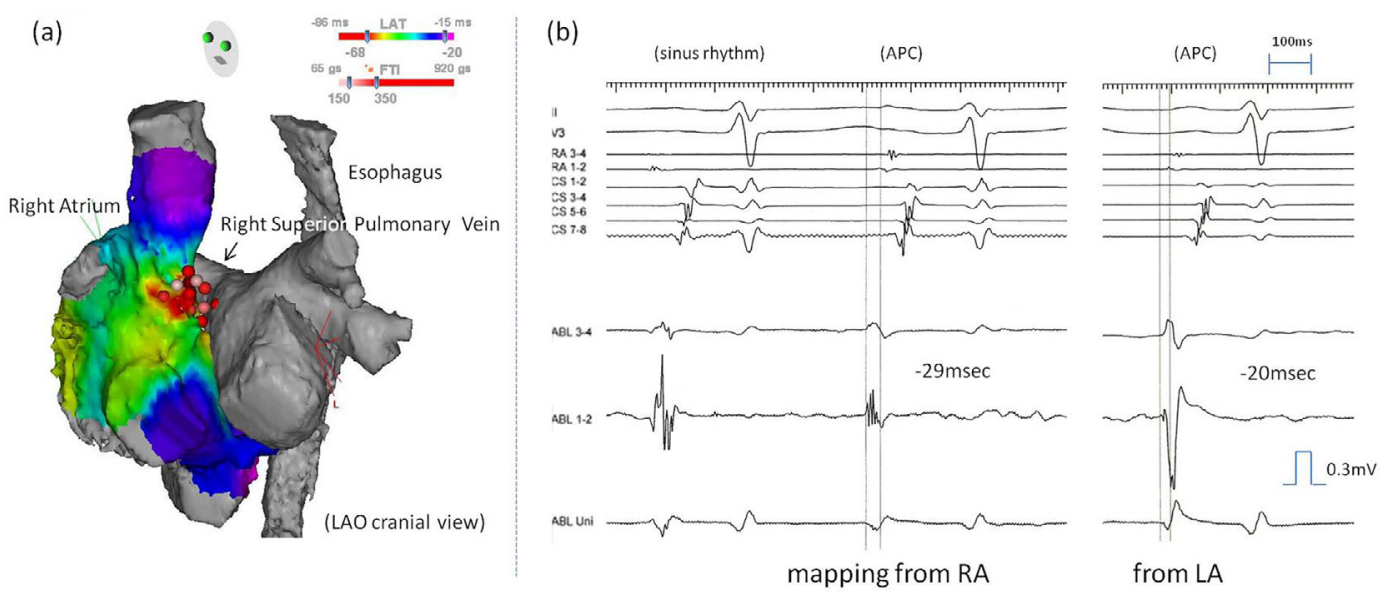

Figure 2. (a) An activation map from the right atrium of the atrial tachycardia with the CARTO3 ${ }^{\circledR}$ system (Biosense Webster, Diamond Bar, USA). The pink and red points indicate the ablation sites. (b) Intracardiac electrocardiograms at the site of the earliest activation during the tachycardia from the high right atrium and left atrium (right superior pulmonary vein ostium).

blocker and pilsicainide, and she was then referred to our institution as a candidate for ablation therapy.

An EPS with a PentaRay ${ }^{\circledR}$ NAV catheter was attempted while the patient swallowed a rice ball. We created an activation map of the RA with a three-dimensional mapping system (CARTO3 ${ }^{\circledR}$ system, Biosense Webster), which revealed that the earliest endocardial breakthrough was on the septal side of the high RA, and the potentials at the earliest site were $29 \mathrm{msec}$ earlier than the beginning of the $\mathrm{P}$ wave. Moreover, the unipolar morphology exhibited a QS pattern (Fig. 2a and b left).

We ablated that site, however, PAT was again detected while eating. Therefore, we approached the LA via a Brockenbrough puncture, and LA mapping was attempted. The earliest site in the LA was at the right superior pulmonary vein (RSPV) ostium, which was adjacent to the earliest site in the RA. This site was not adjacent to the esophagus. The potentials were $20 \mathrm{msec}$ earlier than the beginning of the $\mathrm{P}$ wave, and the unipolar morphology exhibited a $\mathrm{qR}$ pattern (Fig. 2b right).

After ablation at that site (maximum 30W), we confirmed there was no further induction of PAT by swallowing solid food, and the session was ended. The subsequent follow-up has remained uneventful.

\section{Discussion}

We herein report a case of SIAT that was successfully ab- 

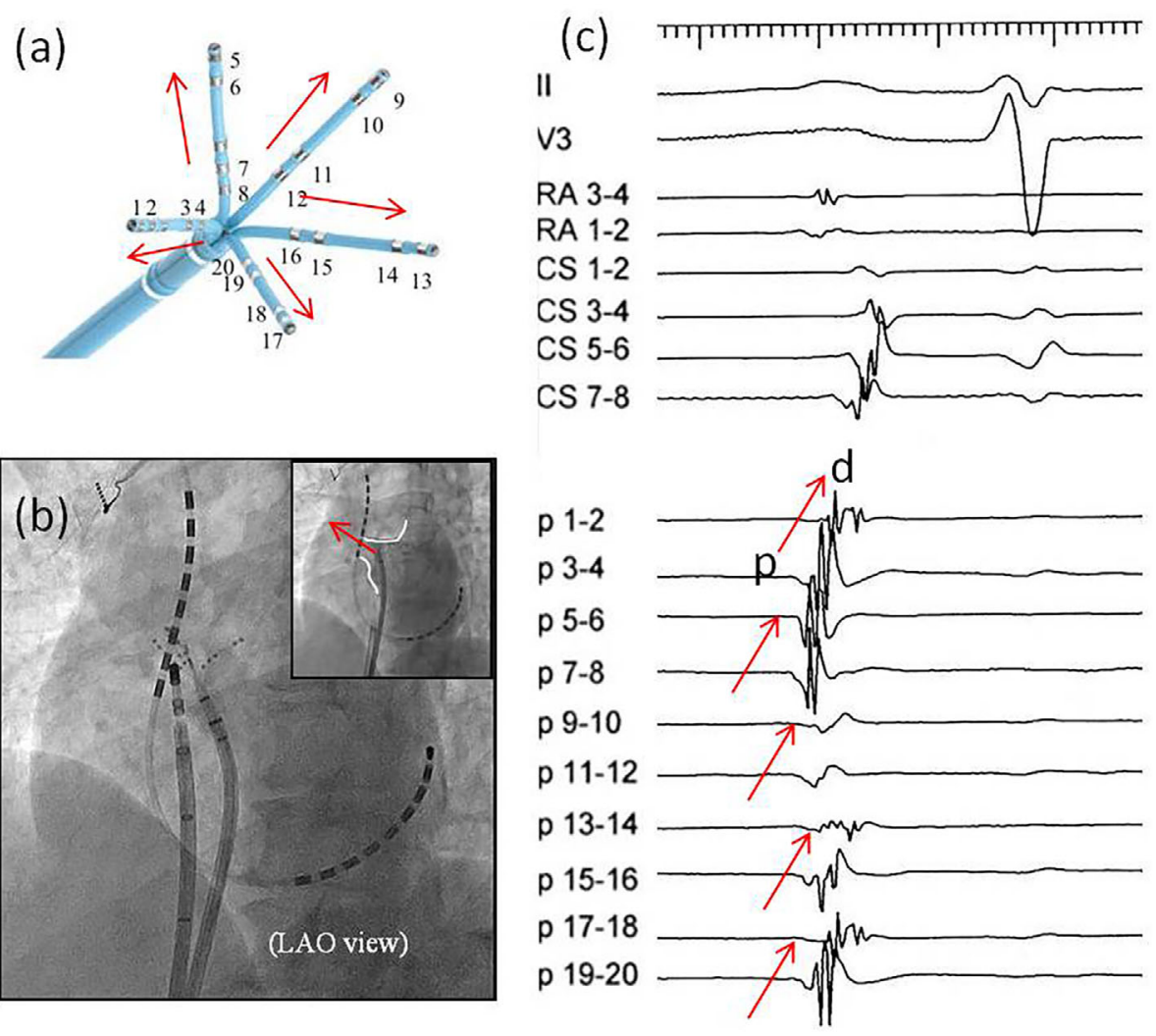

Figure 3. (a) The PentaRay ${ }^{\circledR}$ catheter. This image is modified from the Biosense Webster catalog. (b) A fluoroscopy image in the left anterior oblique (LAO) view. The ablation catheter and PentaRay ${ }^{\circledR}$ catheter were positioned at the site of the earliest activation from the left atrium (LA). In the upper right panel, a schematic diagram of the right superior pulmonary vein ostium is shown. "p," proximal; "d," distal. (c) An intracardiac ECG while mapping with a PentaRay ${ }^{\circledR}$ NAV catheter from the LA. The sequence was from the proximal to distal site of the PentaRay ${ }^{\circledR}$ NAV catheter. Red arrows show the sequence of the tachycardia.

lated under guidance with a unique multipolar electrode catheter, the PentaRay ${ }^{\circledR}$. In this case, there was no induction of PAT by any pacing maneuvers or an intravenous injection of isoproterenol or edrophonium, and PAT was transiently induced only by swallowing solid food. Therefore, to create an activation map, the patient had to frequently eat rice balls.

The efficacy of the radiofrequency catheter ablation (RFCA) for SIAT with a non-contact mapping system was first reported by Tada et al. (7), and in our case, we considered whether to use a non-contact mapping system. However, mapping of a tunnel-like structure on the polar side of the EnSite ${ }^{\mathrm{TM}}$ balloon typically lacks accuracy $(8,9)$. Therefore, it appeared to be difficult to record an accurate site of the tachycardia origin around the right pulmonary vein (RPV) ostium using only the EnSite ${ }^{\mathrm{TM}}$ Array $^{\mathrm{TM}}$ catheter (Endocardial Solutions, St. Jude Medical, St. Paul, USA). Therefore, additional contact mapping might have been required in this situation. The PentaRay ${ }^{\circledR}$ NAV catheter has 20 poles divided among five branches (Fig. 3a). Due to this particular structural feature, we could identify an accurate activation pattern of the tachyarrhythmias with just a few mapping points around the earliest site (10). Therefore, we selected a system with a multipolar mapping catheter (PentaRay ${ }^{\circledR}$ NAV catheter), and the origin of this tachycardia was identified on the high septum, and not from the RPV, because the activation sequence was from the proximal to distal site on the PentaRay ${ }^{\circledR}$ NAV catheter (Fig. 3b and c).

The mechanism of SIAT has not yet been clarified. In the present case, PAT was repetitively observed only when the patient swallowed the upper gastrointestinal endoscope, however, no further arrhythmias were observed when the scope passed through the esophagus. Moreover, esophagography revealed that the earliest site of PAT was not adjacent to the esophagus (Fig. 4). Therefore, mechanical stimulation of the LA by a distended esophagus appeared to be excluded from the potential mechanisms of this SIAT. Thus, SIAT may have occurred due to a vasovagal reflex and/or adrenergic reflex, however, it was not induced by an intravenous injection of isoproterenol or edrophonium. Autonomic reflexes originating in the esophagus might be related to SIAT.

Hojo et al. reported that the mechanism of SIAT might be related to the stimulation of cardiac autonomic ganglionated plexi (GPs) (11). The cardiac GPs are a part of the intrinsic cardiac autonomic nervous system and contain clusters of 


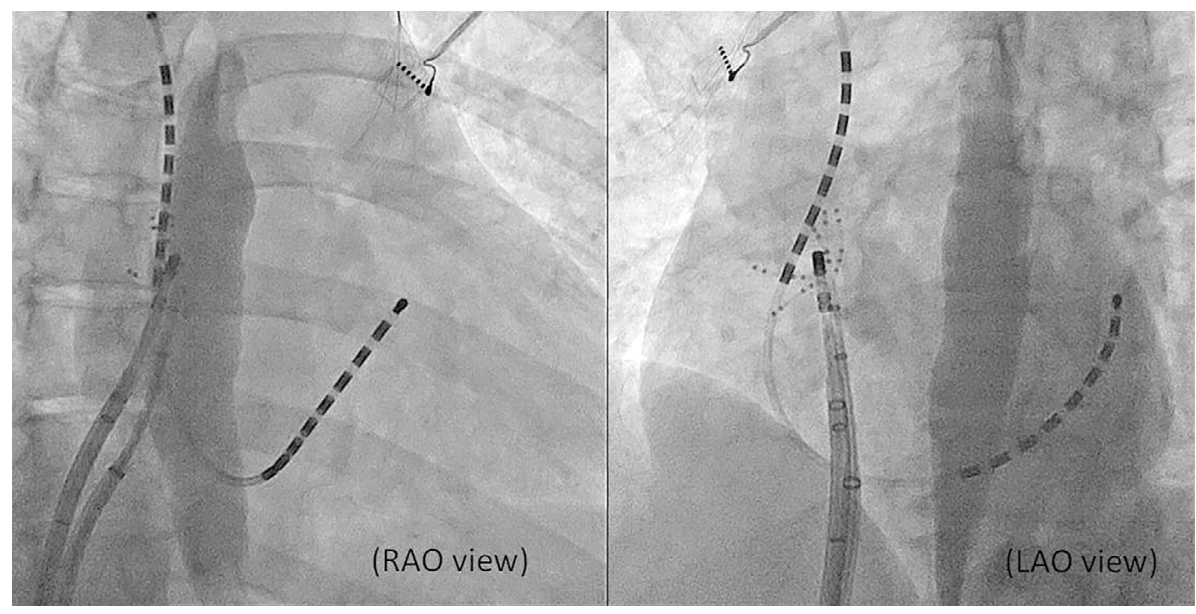

Figure 4. A fluoroscopic image during esophagography. The PentaRay ${ }^{\circledR}$ NAV catheter and ablation catheter are located at the earliest site of the tachycardia on the right atrial side.

autonomic ganglia. In the present case, the earliest site of activation was at the high RA and RSPV ostium, and it was close to the anterior right GP site in the LA (12). There was no change in the blood pressure or heart rate while ablating the anatomical site of the anterior right GP. Before ablation of that site, we did not attempt high frequency stimulation (HFS) of the GP because HFS could induce atrial fibrillation. However, these points are potential limitations in the present case.

The local bipolar potentials on the RA side appeared to be near-field potentials and those on the LA side appeared to be far-field potentials. On the other hand, the local unipolar potentials on the RA side had a QS pattern, while those on the LA side had a $\mathrm{qR}$ pattern (Fig. 2b).

The initial $\mathrm{q}$ wave could be explained as a vector moving forward from this catheter position on the LA septum. We therefore speculated that direct conduction toward the LA remained even after the applications from the RA. Furthermore, repolarization to the hyperpolarization phase of this unipolar electrogram was obviously elevated (ST elevation), which confirms that this region was an adjacent site to the first ablation target site in the RA in this situation.

Finally, the sequential ablation applications from both atria eliminated this tachyarrhythmia. Therefore, we speculated that the origin of this tachyarrhythmia was located on the epicardial tissue between the RA and LA, not inside the RSPV. This epicardial tissue had multiple connections to the septopulmonary bundle of the RPV (13). This complex epicardial connection for the LA might cause a secondary "R" vector of the local unipolar electrogram as a " $q R$ ". However, we concluded that the origin was closer to the RA than LA. Therefore, we might have ablated these epical connection(s) toward the LA and not the tachycardia origin.

We herein experienced successful ablation of SIAT with a PentaRay ${ }^{\circledR}$ NAV catheter. Using this catheter, we could identify the earliest site with only a minimal chance to map short duration tachycardias of even only a few beats, as in our case. We therefore recommend that a PentaRay ${ }^{\circledR}$ NAV catheter should be considered as the first choice for the navigating catheter to map SIAT.

The authors state that they have no Conflict of Interest (COI).

\section{Acknowledgement}

We would like to thank Mr. John Martin for linguistic assistance in the preparation of this manuscript.

\section{References}

1. Tada H, Kaseno K, Kubota S, et al. Swallowing-induced atrial tachyarrhythmias: prevalence, characteristics, and the results of the radiofrequency catheter ablation. PACE 30: 1224-1232, 2007.

2. Bexton RS, Nathan AW, Hellestrand KJ, Camm AJ. Paroxysmal atrial tachycardia provoked by swallowing. Br Med J (Clin Res Ed) 282: 952, 1981.

3. Abinader EG, Cooper M. Swallowing-induced supraventricular tachycardia. Chest 76: 478-480, 1979.

4. Wilmshurst PT. Tachyarrhythmias triggered by swallowing and belching. Heart 81: 313-315, 1999.

5. Hachiya H, Ernst S, Ouyang F, et al. Topographic distribution of focal left atrial tachycardias defined by electrocardiographic and electrophysiological data. Circ J Feb 69: 205-210, 2005.

6. Tang CW, Scheinman MM, Van Hare GF, et al. Use of p wave configuration during atrial tachycardia to predict site of origin. J Am Coll Cardiol 26: 1315-1324, 1995.

7. Tada H, Kaseno K, Naito S, Oshima S. Non-contact threedimensional mapping and ablation of swallowing-induced atrial tachyarrhythmias: two case reports. J Cardiovasc Electrophysiol 18: 1206-1209, 2007.

8. Narita S, Miyamoto K, Tsuchiya T, Nagamoto Y, Yamaguchi T. Radiofrequency catheter ablation of atrial tachycardia under navigation using the EnSite array. Circ J 74: 59-65, 2010.

9. Okishige K, Kawabata M, Umayahara S, et al. Radiofrequency catheter ablation of various kinds of arrhythmias guided by virtual electrograms using a noncontact, computerized mapping system. Circ J 67: 455-460, 2003.

10. Haïssaguerre M, Honcini M, Sanders $P$, et al. Localized sources maintaining atrial fibrillation organized by prior ablation. Circulation 113: 616-625, 2006.

11. Hojo R, Fukamizu S, Ishikawa $T$, et al. Ablation of swallowinginduced atrial tachycardia affects heart rate variability: a case report. Heart Vessels 29: 417-421, 2014. 
12. Nakagawa H, Scherlag BJ, Patterson E, Ikeda A, Lockwood D, Jackman WM. Pathophysiologic basis of autonomic ganglionated plexus ablation in patients with atrial fibrillation. Heart Rhythm 6: S26-S34, 2009.

13. Cabrera JA, Ho SY, Climent V, Fuertes B, Murillo M, Sánchez-Quintana M. Morphological evidence of muscular connections between contiguous pulmonary venous orifices: relevance of the intrapulmonary isthmus for catheter ablation in atrial fibrillation. Heart Rhythm 6: 1192-1198, 2009.

The Internal Medicine is an Open Access article distributed under the Creative Commons Attribution-NonCommercial-NoDerivatives 4.0 International License. To view the details of this license, please visit (https://creativecommons.org/licenses/ by-nc-nd/4.0/).

(C) 2016 The Japanese Society of Internal Medicine http://www.naika.or.jp/imonline/index.html 